Nanoassembly Technique Draws on Lessons from Cell Membranes

E. Zubarev and colleagues at Rice University have discovered how to assemble gold and silver nanoparticle building blocks into larger structures based on the self-assembly of lipid membranes that surround living cells. As reported in the November 29, 2006, issue of the Journal of the American Chemical Society (p. 15098; DOI: $10.1021 / j a 066708 \mathrm{~g}$ ), the method makes use of the hydrophobic effect, a biochemical phenomenon that all living creatures use to create membranes, ultrathin barriers of fatty acids that form a strong yet dynamic sack around the cell, sealing it from the outside world. Cell membranes are one example of a micelle, a strong bilayer covering that is made of two sheets of lipid-based amphiphiles, molecules that have a hydrophilic and a hydrophobic end. Like two pieces of cellophane tape being brought together, the hydrophobic sides of the amphiphilic sheets stick to one another, forming the bilayered micelle.

"When the micelle forms, the process drives the packing of all the junction points, which connect the hydrophobic and the hydrophilic part of an amphiphile, into a high-density array," said Zubarev, the Norman Hackerman-

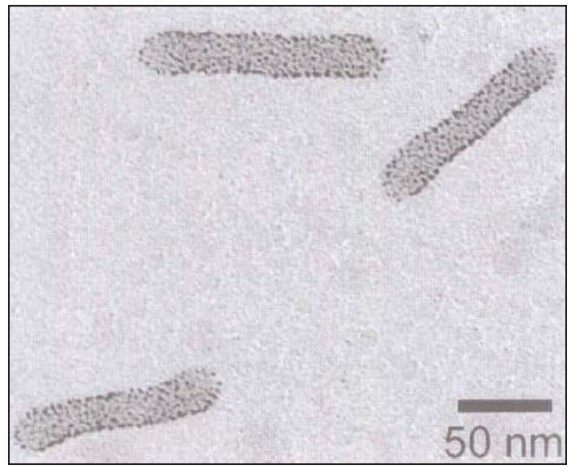

Figure 1. Electron microscope image shows tightly packed cylinders of gold nanoparticles. Note that the edges are darker than the centers, consistent with tubular structures. Credit: Eugene Zubarev/Rice University.

Welch Young Investigator and assistant professor of chemistry. "By attaching a nanoparticle to the junction point of an amphiphile, we can, in effect, use micellization as a means to assemble billions and billions of individual nanoparticles into well-defined one-dimensional superstructures that are soluble in water."

Zubarev and colleagues synthesized V- shaped amphiphiles of polystyrene- $b$ poly(ethylene oxide) and attached 2-nmdiameter gold particles at the focal point of the V. Upon adding water and inducing micelle formation, the team found it could create tightly packed cylinders of gold nanoparticles measuring just $18 \mathrm{~nm}$ in diameter.

All micelles form in three allowable shapes: spheres, cylinders, and sack-like vesicles (see Figure 1). By varying the length of the polystyrene arm, the solvents used, and the size of the gold particles, Zubarev and colleagues were able to form spheres and vesicles and vary the diameter of their cylinders, some of which grew to well over $1000 \mathrm{~nm}$ in length.

"We believe further manipulation of these parameters may provide an opportunity to control the optical and catalytic properties of these nanoassemblies," Zubarev said.

\section{Complex Order Parameter in Ruthenate Superconductors Confirmed}

Since it was discovered to be superconducting over a decade ago, the pairing symmetry of strontium ruthenium oxide has been widely explored and debated. Now, a team of researchers led by D. Van
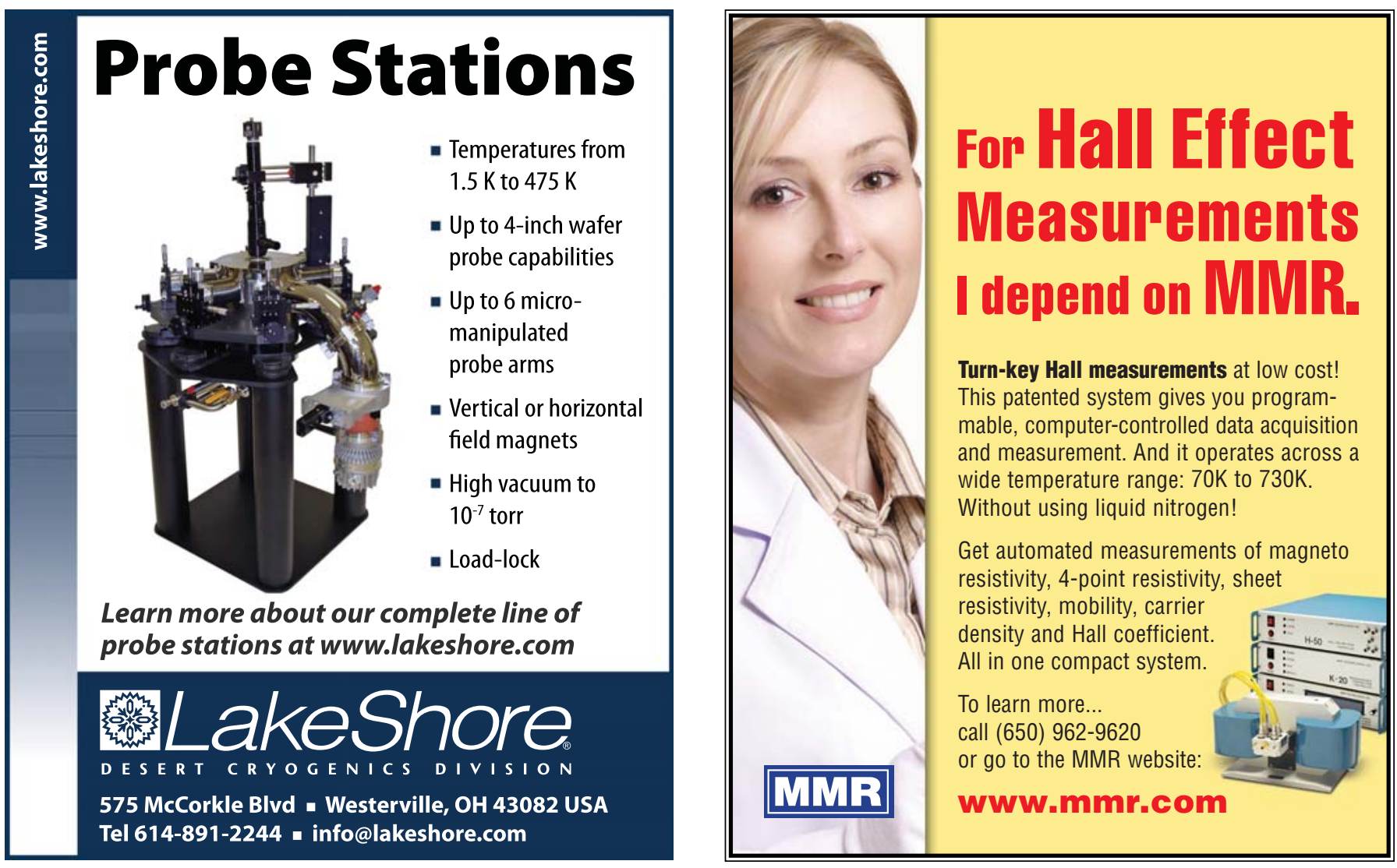
Harlingen at the University of Illinois at Urbana-Champaign have confirmed a complex order parameter in ruthenate superconductors.

"We have pretty unambiguous evidence for ' $p$-wave' symmetry with a complex order parameter that breaks timereversal symmetry in this ruthenate superconductor," said Van Harlingen, a Willett Professor and head of the Department of Physics at Illinois.

Until now, this complex, odd symmetry state had been predicted by theoreticians, but not fully confirmed. Van Harlingen, Y. Maeno of Kyoto University, and their colleagues reported their latest findings in the November 24, 2006, issue of Science (p. 1267).

The order parameter of a superconductor characterizes the nature of the pairing interaction that forms Cooper pairs. It controls many of the superconductor's properties and provides a crucial clue to the microscopic mechanism responsible for the superconductivity.

Conventional superconductors that form Cooper pairs through phonon interactions have an "s-wave" symmetry with an isotropic order parameter. Unconventional superconductors, however, have anisotropy in either or both the phase and magnitude of the order parameter.

Ten years ago, Van Harlingen's group pioneered the Josephson interferometer technique that showed the high-temperature superconducting cuprates had " $d$-wave" symmetry. They are now applying the technique to a wide range of superconducting materials suspected of having unconventional symmetry.

"Our technique can directly measure phase differences in the superconducting order parameter," said Van Harlingen, who is also a member of the Frederick Seitz Materials Research Laboratory and a professor in the university's Center for Advanced Study. "This allows us to make an unambiguous determination of the pairing symmetry in unconventional superconductors," he said.

To use their interferometer technique, the researchers begin by constructing a corner Josephson junction that straddles different faces of a single crystal of the ruthenate superconductor. They then measure the magnetic-field modulation of the supercurrent that reveals the phase shift between different tunneling directions.

If all areas of a Josephson junction have the same order parameter phase, the critical current-measured as a function of applied magnetic field-will create a Fraunhofer diffraction pattern, analogous to a single-slit optical diffraction pattern. However, phase differences in the order parameter on adja- cent crystal faces of a corner junction, or the presence of chiral domains (characterized by the direction of phase winding) along a single junction face, will result in modulated diffraction patterns.

"We observed highly modulated diffraction patterns across single edge junctions, which implies the existence of chiral domains," Van Harlingen said. Abrupt changes seen in the diffraction patterns as a function of magnetic field or time demonstrate that these domains are dynamical, changing their size or orientation.

"The presence of these domains and the distinctly different diffraction patterns observed on orthogonal faces of the same single crystal confirms the ' $p$-wave' triplet spin-pairing state and the complex nature of the superconducting order parameter in the ruthenate superconductors," Van Harlingen said.

\section{Biocatalysts Used to Enable Hydrogen Fuel Cell with No Proton} Exchange Membrane

The use of hydrogen as a clean fuel faces many challenges. One problem is the design of fuel cells, which harness as electricity the energy from the reaction of $\mathrm{H}_{2}$ with $\mathrm{O}_{2}$ more efficiently than does combustion. Typical hydrogen fuel cells necessarily employ a proton-exchange membrane (PEM) to separate the anode (and highly pure $\mathrm{H}_{2}$ ) from the cathode $\left(\right.$ and $\left.\mathrm{O}_{2}\right)$ because the respective electrocatalysts (platinum or platinum with certain other metals) are not selective between $\mathrm{H}_{2}$ and
$\mathrm{O}_{2}$. Highly selective catalysts would make a PEM unnecessary and power could even be generated from very low levels of $\mathrm{H}_{2}$ in air. Recently, F.A. Armstrong, B. Friedrich, and co-researchers at the Inorganic Chemistry Laboratory, University of Oxford, United Kingdom, and the Institute of Biology/Microbiology, Humboldt University of Berlin, Germany, used catalysts found in nature to fabricate without a PEM a fuel cell that generates electricity from just $3 \% \mathrm{H}_{2}$ in air, which is below the combustion limit.

As reported recently in Chemical Communications (p. 5033; DOI: 10.1039/ b614272a), Armstrong, Friedrich, and co-researchers used a graphite anode modified with an unusual bacterial membrane-bound hydrogenase $(\mathrm{MBH})$ and a graphite cathode modified with laccase- - a fungal $\mathrm{O}_{2}$ reductase. Whereas many previously discovered hydrogenases are sensitive to $\mathrm{O}_{2}$, $\mathrm{MBH}$ is very robust and catalytically active under ambient $\mathrm{O}_{2}$ concentrations. The $\mathrm{MBH}$ catalyzes the oxidation of $\mathrm{H}_{2}$ to $2 \mathrm{H}^{+}$, and laccase, which has a high selectivity for $\mathrm{O}_{2}$, catalyzes the reduction of $\mathrm{O}_{2}$ to water. The fuel cell consists of the anode and cathode placed side by side in a tray and covered in a buffer solution, made weakly acidic to optimize both the MBH and laccase reactions.

Containing the fuel cell in a sealed tank allowed the researchers to control the composition of the atmosphere (see Figure 1). With a stationary atmosphere of $3 \% \mathrm{H}_{2}$ in air, the fuel cell gave an open circuit (OC)

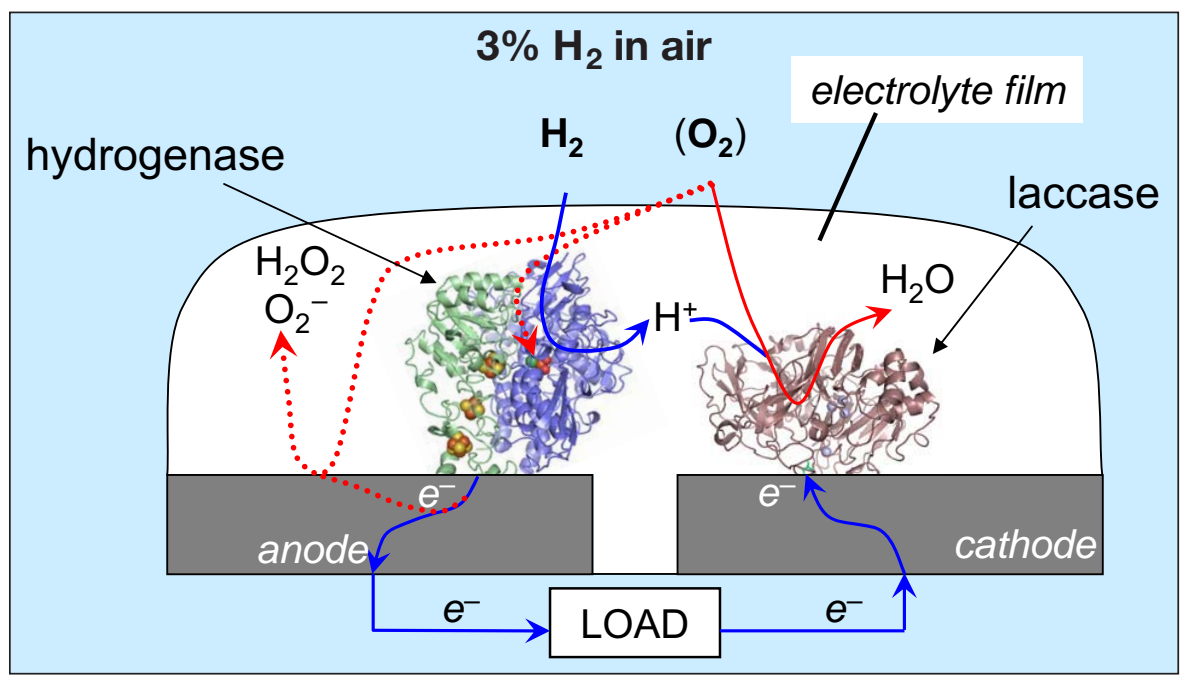

Figure 1. The fuel cell is comprised of a graphite anode modified with $\mathrm{O}_{2}$-tolerant, membrane-bound hydrogenase $(\mathrm{MBH})$ and a graphite cathode modified with high-potential fungal laccase in aqueous electrolyte exposed to an atmosphere of just $3 \% \mathrm{H}_{2}$ in air. The $\mathrm{MBH}$ oxidizes $\mathrm{H}_{2}$ to $\mathrm{H}^{+}$at the anode and must produce sufficient current to compete with nonproductive, that is, short-circuiting, reduction of $\mathrm{O}_{2}$ at bare areas of the electrode. In addition, the $\mathrm{MBH}$ must exhibit tolerance to inhibition and damage by $\mathrm{O}_{2}$ itself and by the reactive species $\mathrm{O}_{2}^{-}$and $\mathrm{H}_{2} \mathrm{O}_{2}$, which are produced by the direct reduction of $\mathrm{O}_{2}$. 